\title{
Effects of ulinastatin on coagulation in high-risk patients undergoing off-pump coronary artery bypass graft surgery
}

\author{
Na-Young Kim ${ }^{1}$, Jae-Kwang Shim ${ }^{1,2}$, Seo-Ouk Bang ${ }^{1,2}$, Jee-Suk Sim ${ }^{1}$, Jong-Wook Song ${ }^{1,2}$, and \\ Young-Lan Kwak ${ }^{1,2}$ \\ ${ }^{1}$ Department of Anesthesiology and Pain Medicine, ${ }^{2}$ Anesthesia and Pain Research Institute, Yonsei University College of Medicine, \\ Seoul, Korea
}

Background: Both systemic inflammatory reaction and regional myocardial ischemia/reperfusion injury may elicit hypercoagulability after off-pump coronary artery bypass grafting (OPCAB). We investigated the influence of ulinastatin, which suppresses the activity of polymorphonuclear leukocyte elastase and production of proinflammatory cytokines, on coagulation in patients with elevated high-sensitivity C-reactive protein (hsCRP) undergoing OPCAB.

Methods: Fifty patients whose preoperative hsCRP $>3.0 \mathrm{mg} / \mathrm{L}$ were randomly allocated into the ulinastatin (600,000 $\mathrm{U})$ or control group. Serum concentrations of thrombin-antithrombin complex (TAT) and prothrombin fragment 1+2 $(\mathrm{F} 1+2)$ were measured preoperatively, immediately after surgery, and at $24 \mathrm{~h}$ after surgery, respectively. Secondary endpoints included platelet factor (PF)-4, amount of blood loss, and transfusion requirement.

Results: All baseline values of TAT, F1+2, and PF- 4 were higher than the normal range in both groups. F1+2 was elevated in both groups at immediate, and at $24 \mathrm{~h}$ after surgery as compared to baseline value, without any significant intergroup differences. Remaining coagulation parameters, transfusion requirement and blood loss during operation and postoperative $24 \mathrm{~h}$ were not different between the two groups.

Conclusions: Intraoperative administration of ulinastatin did not convey beneficial influence in terms of coagulation and blood loss in high-risk patients with elevated hsCRP undergoing multivessel OPCAB, who already exhibited hypercoagulability before surgery. (Korean J Anesthesiol 2013; 64: 105-111)

Key Words: Coagulability, hsCRP, OPCAB, Ulinastatin.

Received: February 7, 2012. Revised: 1st, May 25, 2012; 2nd, July 19, 2012. Accepted: August 24, 2012.

Corresponding author: Jong-Wook Song, M.D., Department of Anesthesiology and Pain Medicine and Anesthesia and Pain Research Institute, Yonsei University College of Medicine, Severance Biomedical Science Institute, Yonsei University Health System, 250, Seongsan-no, Seodaemun-gu, Seoul 120-752, Korea. Tel: 82-2-2228-8514, Fax: 82-2-364-2951, E-mail: sjw72331@yahoo.co.kr

(c) This is an open-access article distributed under the terms of the Creative Commons Attribution Non-Commercial License (http:// creativecommons.org/licenses/by-nc/3.0/), which permits unrestricted non-commercial use, distribution, and reproduction in any medium, provided the original work is properly cited. 


\section{Introduction}

Despite avoiding cardiopulmonary bypass (CPB) and global myocardial ischemia, a considerable degree of systemic inflammatory reaction still develops during off-pump coronary artery bypass grafting (OPCAB) [1]. In addition, various degree of cumulative regional warm ischemia/reperfusion (I/R) injury occurs following multivessel grafting. Of concern, regional warm I/R injury predisposed patients to increased thrombin formation and hypercoagulable state in previous studies, which may be aggravated by the accompanying systemic inflammatory response [2]. In $\mathrm{OPCAB}$, hypercoagulable state during the perioperative period may draw particular attention in terms of decreased graft patency and development of major adverse cardiac events (MACE) as compared to conventional on-pump coronary artery bypass grafting (CABG) [3].

Ulinastatin is a glycoprotein extracted and purified from human urine. It suppresses the activity of polymorphonuclear leukocyte elastase (PMNE) [4], and has been reported to decrease systemic inflammatory response following CPB [5]. Furthermore, in view of coagulation, it inhibited coagulation and fibrinolysis following major abdominal surgery [6].

Under recognition of the central role of inflammation in coronary artery occlusive disease (CAOD), high sensitivity C-reactive protein (hsCRP), an acute phase reactant in inflammation, has been regarded as an important predictor of poor postoperative outcome as well as cardiovascular risk in CAOD patients $[7,8]$. Inflammation contributes to the thrombotic response and influences the initiation and propagation of blood coagulation [9], and CRP was reported to directly influence thrombin generation and/or coagulation activation [10]. Therefore, patients with elevated preoperative hsCRP undergoing OPCAB may be more prone to develop a hypercoagulable state during the perioperative period, while studies validating the efficacy for preventive measures in this subset of patients are lacking.

In the present study, we designed this prospective single-site, double-blinded, randomized, and controlled trial to investigate the effect of ulinastatin on coagulation system, especially regarding the markers of thrombin formation, in patients with elevated preoperative hsCRP undergoing multivessel OPCAB.

\section{Materials and Methods}

After obtaining approval from our Institutional Review Board and written informed consent from the patients, 50 patients scheduled for elective OPCAB were enrolled for the present study. The inclusion criteria were patients in whom preoperative hsCRP measured one day before the operation was greater than $3.0 \mathrm{mg} / \mathrm{L}$ [7]. Patients were excluded in case of salvage and/ or emergency operation, preoperative serum creatinine $>1.4$ $\mathrm{mg} / \mathrm{dl}$, liver disease, preoperative heparinization or preexisting coagulation disorder. Patients who met the inclusion criteria were randomized into the ulinastatin or control group according to computer-generated codes. The group assignment for each patient was sealed in sequentially numbered, opaque envelopes. A nurse who was not involved in the anesthesia and postoperative care of the patients opened these envelopes and prepared ulinastatin or saline solution according to the group assignment to ensure double-blindness.

Primary end point of the current study was to serially compare serum concentrations of thrombin-antithrombin complex (TAT) and prothrombin fragment $1+2(\mathrm{~F} 1+2)$ between the groups, which are well-known indices of in vivo thrombin generation [11]. Secondary end points of this study were to compare differences in other coagulation parameters including platelet factor (PF)-4, amount of postoperative blood loss, and the incidence of postoperative myocardial infarction (MI).

Patients were premedicated with $0.05-0.1 \mathrm{mg} / \mathrm{kg}$ of intramuscular morphine $1 \mathrm{~h}$ before the operation. Standard monitoring devices were applied to the patients on arrival at the operating room, including 5-lead electrocardiogram, pulse oximetry, a radial artery catheter and a pulmonary artery catheter (PAC, Swan-Ganz Combo CCO/SvO ${ }_{2}$, Edwards Lifesciences LLC, USA). PAC was inserted through the right internal jugular vein and connected to an analysis system (Vigilance, Edwards Lifesciences LLC, USA) for continuous monitoring of the cardiac index and mixed venous oxygen saturation $\left(\mathrm{SvO}_{2}\right)$. Anesthesia was induced with intravenous midazolam (0.03-0.07 mg/kg), sufentanil (1.5-2.0 $\mu \mathrm{g} / \mathrm{kg})$ and rocuronium ( $0.9 \mathrm{mg} / \mathrm{kg})$. Patients' lungs were mechanically ventilated with a tidal volume of $7-8 \mathrm{mg} / \mathrm{kg}$ and a positive endexpiratory pressure of $5 \mathrm{cmH}_{2} \mathrm{O}$ at a rate of $8-12$ breaths/min to maintain normocarbia. Anesthesia was maintained with $0.8-1.5 \%$ of sevoflurane in a $40 \%$ oxygen/air mixture and a continuous infusion of sufentanil $(0.5-1.5 \mu \mathrm{g} / \mathrm{kg} / \mathrm{h})$.

In the ulinastatin group, 300,000 $\mathrm{U}$ of intravenous ulinastatin was administered after anesthetic induction and at the onset of Y-graft construction, respectively. In the control group, equal volume of saline placebo was administered at each corresponding time points.

After a median sternotomy, the heart was displaced using posterior pericardial stitches, large gauze swabs and tissue stabilizer (Octopus, Medtronic Inc., USA). During the period of heart displacement, crystalloid solution was infused at a fixed rate of $6 \mathrm{ml} / \mathrm{kg} / \mathrm{h}$, and colloid solution was administered according to the estimated blood loss. The blood salvaged by the cell salvage device was re-infused to the patient after sternum closure. During the period of heart displacement and grafting, hypotension (mean systemic arterial pressure below $70 \mathrm{mmHg}$ ) was managed using either a $5-10^{\circ}$ Trendelenburg position 
or a norepinephrine infusion. Intravenous milrinone was infused at $0.5 \mu \mathrm{g} / \mathrm{kg} / \mathrm{min}$ if a $\mathrm{SvO}_{2}<60 \%$ for more than $10 \mathrm{~min}$ or mitral regurgitation $\geq$ grade 3 developed with a concomitant increase in the mean pulmonary arterial pressure $>30 \mathrm{mmHg}$. Allogeneic packed red blood cells (pRBCs) were transfused to maintain the hematocrit (Hct) $>25 \%$ for patients aged more than 65 years and $>22 \%$ for patients aged less than 65 years throughout the study period. The blood temperature measured by a PAC was maintained above $36^{\circ} \mathrm{C}$ with a warm mattress, a forced warm air blanket and a fluid warmer as necessary.

For intraoperative anticoagulation, intravenous heparin (100 U/kg) was administered at the completion of left internal mammary artery harvesting. Activated clotting time (ACT) was measured 5 min after the initial administration of heparin and repeated at an interval of $30 \mathrm{~min}$ thereafter until the completion of grafting. Additional 2,000 U of heparin was administered if the ACT was less than $250 \mathrm{~s}$. Anticoagulation was reversed with $0.5 \mathrm{mg}$ of protamine per $100 \mathrm{U}$ of heparin.

All the patients were transferred to the intensive care unit (ICU) after the surgery. The ICU staffs who were not aware of the study groups conducted the postoperative care of the patients, including decisions for hemodynamic management, transfusion, extubation and discharge from the ICU according to the standard ICU protocols of our institution. Postoperative antiplatelet therapy comprising of $100 \mathrm{mg}$ of aspirin and 75 mg of PO clopidogrel once a day, was started from the evening of the surgery, unless contraindicated. None of the patients received postoperative heparinization during the study period.

Blood samples for the measurements of TAT, F1+2, PF4, prothrombin time (PT), activated partial thromboplastin time (aPTT), hsCRP, white blood cell (WBC) count, neutrophil count, hematocrit, platelet count, creatine kinase (CK)-MB, and troponin ( $\mathrm{Tn}$ )-T were obtained preoperatively, immediately after surgery, and $24 \mathrm{~h}$ after surgery. Fluid balance, amount of blood loss, and transfusion requirement during operation and postoperative $24 \mathrm{~h}$ were also recorded. Postoperative MI was defined as the occurrence of an increase in Tn-T or CKMB more than 5 times the upper normal limit when associated with the development of new pathologic Q-wave or left bundle branch block on the electrocardiogram.

Based on a previous study [6], 22 patients were required in each group for $90 \%$ power to detect a $20 \mathrm{ng} / \mathrm{ml}$ difference in the TAT between the groups with a SD of $20 \mathrm{ng} / \mathrm{ml}$ at an $\alpha$ value of 0.05 . The study size was set as 25 patients in each group considering a dropout rate of $10 \%$. All data are expressed as mean \pm SD or median (IQR) or the number of patients. The data were analyzed using $\chi^{2}$ tests, Fisher's exact test, independent t-tests or Wilcoxon signed rank tests as appropriate. The repeatedly obtained data were analyzed by repeated measures of ANOVA or Friedman tests according to the normality of distribution.
Post-hoc tests were conducted with Bonferroni correction. The statistical analyses were performed using SAS 9.1.3 (Institute Inc., Cary, NC, USA). A P value of less than 0.05 was considered statistically significant.

\section{Results}

OPCAB was successfully performed in all 50 patients. Preoperative characteristics of the patients were similar between the two groups (Table 1).

Coagulation parameters including TAT, F1+2, and PF-4 are shown in Table 2. There were no differences in TAT, F1+2, PF-4 PT, and aPTT between the groups throughout the study period. Baseline values of TAT, F1+2, and PF-4 were all above the normal reference range in both groups. F1+2 increased immediately after surgery $(\mathrm{P}=.005$ and $\mathrm{P}<.001$ for the control and ulinastatin group, respectively) and $24 \mathrm{~h}$ after surgery $(\mathrm{P}=.031$ and $\mathrm{P}<.001$ for the control and ulinastatin group, respectively) in both groups as compared to individual corresponding baseline value. No change in the values of TAT and PF-4 was observed throughout the study period as compared to each corresponding baseline value.

There were no significant differences in hematologic parameters, hsCRP, and cardiac enzymes between the groups throughout the study period (Table 3 ). A decrease in HsCRP was observed immediately after surgery $(\mathrm{P}<.05)$ and an increase was noted at $24 \mathrm{~h}$ after surgery without any difference between

Table 1. Patients' Characteristics

\begin{tabular}{lcc}
\hline & $\begin{array}{c}\text { Control } \\
(\mathrm{n}=25)\end{array}$ & $\begin{array}{c}\text { Ulinastatin } \\
(\mathrm{n}=25)\end{array}$ \\
\hline Sex (M/F) & $17 / 8$ & $19 / 6$ \\
Age (yr) & $63 \pm 9$ & $67 \pm 10$ \\
Height (cm) & $160 \pm 7$ & $165 \pm 10$ \\
Weight (kg) & $62 \pm 8$ & $66 \pm 11$ \\
EF (\%) & $55 \pm 19$ & $60 \pm 13$ \\
EuroSCORE & $4.5(2-6)$ & $3(1-5)$ \\
Diabetes mellitus & $11(46 \%)$ & $16(64 \%)$ \\
Hypertension & $15(60 \%)$ & $20(83 \%)$ \\
Preoperative medication & $18(72 \%)$ & $18(72 \%)$ \\
$\quad$ Statin & $20(80 \%)$ & $15(63 \%)$ \\
Aspirin & $15(60 \%)$ & $12(48 \%)$ \\
Clopidogrel & $11(44 \%)$ & $15(63 \%)$ \\
Beta blocker & $13(52 \%)$ & $14(58 \%)$ \\
Calcium channel blocker & $7(28 \%)$ & $9(36 \%)$ \\
ACE inhibitor & $1.9 \pm 1.5$ & $2.2 \pm 1.5$ \\
Aspirin discontinuation (days) & $2.2 \pm 1.7$ & $2.7 \pm 1.7$ \\
Clopidogrel discontinuation (days) & $91.1 \pm 13.6$ & $88.8 \pm 16.7$ \\
Antithrombin III (\%) & $415 \pm 94$ & $418 \pm 98$ \\
Fibrinogen (mg/dl) &
\end{tabular}

Values are mean \pm standard deviation or median (interquartile range) or number of patients (percentage). EF: left ventricular ejection fraction, ACE inhibitor: angiotensin converting enzyme inhibitor. There were no significant differences between the two groups. 
Table 2. Parameters Related to Coagulation

\begin{tabular}{llccc}
\hline & & Preoperative & Immediate after surgery & $24 \mathrm{~h}$ after surgery \\
\hline TAT $(\mathrm{ng} / \mathrm{ml})$ & Control & $30.3(23.1-49.9)$ & $27.6(13.5-53.0)$ & $29.1(17.2-41.8)$ \\
& Ulinastatin & $26.0(18.1-44.3)$ & $38.7(18.5-51.1)$ & $30.4(18.4-43.8)$ \\
F1+2 (nmol/L) & Control & $1.06(0.58-1.84)$ & $2.54(1.02-4.15)^{*}$ & $3.29(1.05-6.49)^{*}$ \\
& Ulinastatin & $1.09(0.55-3.53)$ & $4.54(1.73-6.90)^{*}$ & $3.75(1.12-7.36)^{*}$ \\
PF-4 (IU/ml) & Control & $19.2 \pm 1.4$ & $18.8 \pm 2.2$ & $18.7 \pm 1.7$ \\
& Ulinastatin & $19.0 \pm 1.3$ & $19.2 \pm 1.0$ & $18.3 \pm 2.5$ \\
PT (s) & Control & $11.2 \pm 0.6$ & $12.8 \pm 1.2^{*}$ & $12.7 \pm 1.0^{*}$ \\
aPTT (s) & Ulinastatin & $10.9 \pm 0.8$ & $12.7 \pm 1.2^{*}$ & $12.6 \pm 1.0^{*}$ \\
& Control & $32.9 \pm 9.6$ & $31.3 \pm 4.8$ & $31.2 \pm 8.5$ \\
\hline
\end{tabular}

Values are mean \pm standard deviation or median (interquartile range). TAT: thrombin-antithrombin complex, F1+2: prothrombin fragment 1+2, PF-4: platelet factor-4, PT: prothrombin time, aPTT: activated partial thromboplastin time. ${ }^{*} \mathrm{P}<0.05$ vs preoperative value.

Table 3. Hematologic Parameters, hsCRP and Cardiac Enzymes

\begin{tabular}{|c|c|c|}
\hline & Control & Ulinastatin \\
\hline \multicolumn{3}{|l|}{ hsCRP (mg/L) } \\
\hline Preoperative & $7.6(4.0-27.9)$ & $11.2(4.5-15.8)$ \\
\hline Immediate after surgery & $6.1(2.6-13.5) *$ & $5.2(2.5-9.7) *$ \\
\hline $24 \mathrm{~h}$ after surgery & $89.7(70.2-119.0) *$ & $97.7(78.1-125.0) *$ \\
\hline \multicolumn{3}{|l|}{$\mathrm{WBC}\left(/ \mathrm{mm}^{3}\right)$} \\
\hline Preoperative & $7,383 \pm 1,731$ & $7,706 \pm 1,918$ \\
\hline Immediate after surgery & $10,209 \pm 2,780 *$ & $10,966 \pm 2,813^{*}$ \\
\hline $24 \mathrm{~h}$ after surgery & $12,859 \pm 3,145^{*}$ & $13,320 \pm 3,220 *$ \\
\hline \multicolumn{3}{|l|}{ Neutrophil (\%) } \\
\hline Preoperative & $59 \pm 10$ & $60 \pm 11$ \\
\hline Immediate after surgery & $80 \pm 9 *$ & $82 \pm 6^{*}$ \\
\hline $24 \mathrm{~h}$ after surgery & $85 \pm 8^{*}$ & $85 \pm 5^{*}$ \\
\hline \multicolumn{3}{|l|}{ Hematocrit (\%) } \\
\hline Preoperative & $37 \pm 7$ & $37 \pm 5$ \\
\hline Immediate after surgery & $28 \pm 3^{*}$ & $28 \pm 3^{*}$ \\
\hline $24 \mathrm{~h}$ after surgery & $27 \pm 5^{*}$ & $28 \pm 3^{*}$ \\
\hline \multicolumn{3}{|l|}{ Platelet $\left(10^{3} / \mathrm{mm}^{3}\right)$} \\
\hline Preoperative & $230 \pm 92$ & $248 \pm 50$ \\
\hline Immediate after surgery & $173 \pm 66^{*}$ & $176 \pm 55^{*}$ \\
\hline $24 \mathrm{~h}$ after surgery & $173 \pm 68 *$ & $186 \pm 46^{*}$ \\
\hline \multicolumn{3}{|l|}{ CK-MB (ng/ml) } \\
\hline Preoperative & $2.0(1.5-2.5)$ & $2.2(1.6-2.9)$ \\
\hline Immediate after surgery & $4.3(3.7-4.9)^{*}$ & $4.0(3.6-6.4)^{*}$ \\
\hline $24 \mathrm{~h}$ after surgery & $8.3(5.2-13.6)^{*}$ & $5.5(4.5-7.9)^{*}$ \\
\hline \multicolumn{3}{|l|}{$\mathrm{Tn}-\mathrm{T}(\mathrm{ng} / \mathrm{ml})$} \\
\hline Preoperative & $0.013(0.010-0.093)$ & $0.010(0.010-0.152)$ \\
\hline Immediate after surgery & $0.087(0.040-0.133)^{*}$ & $0.111(0.061-0.210) *$ \\
\hline $24 \mathrm{~h}$ after surgery & $0.147(0.078-0.246)^{*}$ & $0.159(0.079-0.245) *$ \\
\hline
\end{tabular}

Values are mean \pm standard deviation or median (interquartile range). hsCRP: high sensitivity C-reactive protein, WBC: white blood cell, CKMB: creatine kinase-MB, Tn-T: Troponin-T. ${ }^{*} \mathrm{P}<0.05$ vspreoperative value.

the groups $(\mathrm{P}<.001$, Table 3$)$.

There were no differences in parameters of intraoperative anticoagulation including initial ACT, amount of heparin injected, mean, minimum and maximum ACT during the operation, amount of protamine for heparin reversal and final ACT between the groups (Table 4).

Fluid balance and amount of blood loss during the operation
Table 4. Parameters of Intraoperative Anticoagulation

\begin{tabular}{lcc}
\hline & $\begin{array}{c}\text { Control } \\
(\mathrm{n}=25)\end{array}$ & $\begin{array}{c}\text { Ulinastatin } \\
(\mathrm{n}=25)\end{array}$ \\
\hline Initial ACT (s) & $141 \pm 15$ & $148 \pm 17$ \\
Dose of heparin (U) & $6,714 \pm 1,443$ & $6,414 \pm 1,489$ \\
Mean ACT during grafting (s) & $290 \pm 58$ & $277 \pm 41$ \\
Minimum ACT during grafting (s) & $242 \pm 42$ & $241 \pm 40$ \\
Maximum ACT during grafting (s) & $342 \pm 91$ & $329 \pm 66$ \\
Dose of protamine (mg) & $33 \pm 12$ & $28 \pm 11$ \\
Final ACT (s) & $156 \pm 15$ & $152 \pm 24$
\end{tabular}

Values are mean \pm standard deviation. ACT: activated clotting time. There were no significant differences between the two groups.

Table 5. Amount of Blood Loss, Fluid Balance and Transfusion Requirement during the Operation and Postoperative $24 \mathrm{~h}$

\begin{tabular}{lcc}
\hline & $\begin{array}{c}\text { Control } \\
(\mathrm{n}=25)\end{array}$ & $\begin{array}{c}\text { Ulinastatin } \\
(\mathrm{n}=25)\end{array}$ \\
\hline Intraoperative & & \\
Crystalloid (ml) & $2,050 \pm 619$ & $2,224 \pm 390$ \\
Colloid (ml) & $1,037 \pm 277$ & $1,062 \pm 286$ \\
Urine output (ml) & $461 \pm 443$ & $557 \pm 381$ \\
Blood loss (ml) & $286 \pm 183$ & $285 \pm 202$ \\
pRBC transfusion (unit) & $0(0-2)$ & $0(0-2)$ \\
Number of patients who required pRBC & $10(40 \%)$ & $7(28 \%)$ \\
Postoperative 24 h & & \\
Crystalloid (ml) & $3,904 \pm 741$ & $3,592 \pm 1,059$ \\
Colloid (ml) & $353 \pm 244$ & $280 \pm 310$ \\
Urine output (ml) & $2,991 \pm 760$ & $2,953 \pm 675$ \\
Blood loss (ml) & $632 \pm 206$ & $571 \pm 160$ \\
pRBC transfusion (unit) & $0(0-2)$ & $0(0-1)$ \\
Number of patients who required pRBC & $4(16 \%)$ & $2(8 \%)$ \\
\hline
\end{tabular}

Values are mean \pm standard deviation or median (range) or number of patients (percentage). pRBC: packed red blood cell. There were no significant differences between the two groups.

and postoperative $24 \mathrm{~h}$ were similar between the groups. Requirement for pRBC transfusion during the operation and postoperative $24 \mathrm{~h}$ were also similar between the groups (Table 5). 10 patients in control group and 7 patients in ulinastatin group were transfused respectively and all the patients who were 
transfused were over 65 years old except one patient in control group.

There were no differences in the duration of ICU stay (3 [34] days vs. 3 [3-4] days for the control and ulinastatin group, respectively, $\mathrm{P}=.206$ ) and postoperative hospitalization (11 [9-18] days vs. 9 [9-11] days for the control and ulinastatin group, respectively, $\mathrm{P}=.234$ ) between the groups. In addition, the incidence of postoperative MI during hospitalization was not different between the two groups ( 0 vs. 1 in the control and ulinastatin group, respectively, $\mathrm{P}=1.000$ ).

\section{Discussion}

In this prospective, double-blinded, randomized, and controlled study addressing the effect of ulinastatin on coagulation in patients whose preoperative hsCRP $>3 \mathrm{mg} / \mathrm{L}$ and were undergoing multivessel $\mathrm{OPCAB}$, we could not observe any beneficial effects of ulinastatin in terms of mitigating development of postoperative hypercoagulable state.

Ulinastatin, a glycoprotein extracted and purified from human urine, inhibits PMNE activity [4] and production of pro-inflammatory cytokines $[12,13]$. Thus, it has been used in the case of patients with inflammatory disorders such as pancreatitis, shock and disseminated intravascular coagulation [14].

Ulinastatin has also been reported to decrease systemic inflammatory response following CPB [5] or trauma with hemorrhagic shock [15]. Furthermore, in view of coagulation, ulinastatin has been reported to normalize the coagulation function during liver resection [16], and inhibit coagulation and fibrinolysis following major abdominal surgery [6].

In hemostatic mechanism, PMNE activity can influence coagulation by modulating various steps. Cleavage of thrombomodulin from the endothelial cell surface by PMNE generates a much less active form of thrombomodulin [17], which could lead to inhibition of protein $\mathrm{C}$ pathway and procoagulant state [9]. PMNE is also known to degrade antithrombin [18]. On the other hand, PMNE enhances fibrinolysis by causing degradation of cross-linked fibrin [19]. TNF suppresses transcription of the thrombomodulin gene in endothelial cells and may affect coagulation [20]. Indeed, ulinastatin has been reported to decrease the levels of PMNE, TNF- $\alpha$, IL-6 and IL-8 after CPB [9]. Not surprisingly, ulinastatin administrated before CPB attenuated the elevation of IL- 6 and IL-8, increased stroke volume index, and decreased intrapulmonary shunt after CPB [21]. In view of coagulation, ulinastatin $(6,000 \mathrm{U} / \mathrm{kg})$ attenuated postoperative increase in TAT and fibrin degradation product (FDP) in abdominal surgery, suggesting inhibitory effect of ulinastatin on coagulation and fibrinolysis associated with major surgery [6]. However, evidence is limited regarding the effect of ulinastatin on coagulation during perioperative period in cardiac surgery.

In conventional on-pump surgery, a relatively low incidence of postoperative thrombotic events was supposed to be attributed in part to an unidentified anticoagulating effect of CPB [22]. On the contrary, OPCAB can lead to a postoperative hypercoagulable state by circumventing CPB [23]. In addition, cumulative regional warm I/R may potentially contribute to postoperative thrombotic complications. Inflammation also affects coagulation cascades in favor of thrombosis through cellular and humoral modulation. Inflammatory cytokines initiate coagulation through the induction of tissue factor expression, primarily on monocyte/macrophages [9]. Plasma hsCRP reflect chronic low-grade inflammatory conditions in patients without apparent inflammation, for example, diabetes mellitus, osteoarthritis and pulmonary disease [24]. In addition, the recognition of inflammatory mechanism in pathophysiology of atherosclerosis has drawn attention on the role of hsCRP as a predictor of cardiovascular events [7]. Elevated preoperative hsCRP has been reported to be associated with adverse postoperative outcome [8], and higher risk of early graft occlusion after CABG [25]. Furthermore, a published report states that CRP directly influenced thrombin generation and/ or coagulation activation in patients with stable angina [10]. Therefore, in patients with elevated levels of hsCRP, it may be reasonable to take concerns regarding the high-risk of developing a hypercoagulable state during the perioperative period, which may potentially increase the risk of ischemic events and endanger the patency of coronary anastomoses. In the current study, thus, we evaluated whether ulinastatin could attenuate the development of hypercoagulability in these subset of patients undergoing multivessel OPCAB.

In the current trial, unfortunately, we could not observe any significant influence of ulinastatin on postoperative TAT, F1+2 and PF-4 levels, which are well known markers of thrombin and platelet activation. $\mathrm{F} 1+2$ is a polypeptide released from prothrombin during its activation to form thrombin by the prothrombinase complex, and subsequently thrombin is inhibited by the formation of TAT by antithrombin III. Thus, $\mathrm{F} 1+2$ and TAT have been generally used as indices of in vivo thrombin generation [11]. In this study, F1+2 measured immediately and $24 \mathrm{~h}$ after surgery were elevated from its preoperative value in both groups, indicating increased thrombin generation. Elevated levels of $\mathrm{F} 1+2$ after OPCAB has also been reported in previous studies $[2,23]$ and could be a possible target for prevention of procoagulant changes during perioperative period. Although ulinastatin has failed to attenuate increased perioperative thrombin generation, there is a report stating that aprotinin, a serine protease inhibitor, could abrogate hypercoagulable state after OPCAB [3]. PF-4 is a platelet $\alpha$-granule CXC chemokine that is released during 
platelet activation. It plays important physiologic roles in the processes of coagulation and excess amount of PF-4 can facilitate thrombosis by bringing together platelets and the endothelium and also by activating monocytes, which carry tissue factor [26]. The values of PF-4 in this study also did not change significantly from the preoperative value in both groups. Significant perioperative platelet activation does not seem to occur after OPCAB at least until postoperative $24 \mathrm{~h}$, as shown in previous studies, where platelet function was evaluated using thromboelastography, whole blood aggregometry or platelet function analyzer-100 [2].

Of interesting to note in the current study is the preoperatively elevated values of TAT, F1+2 and PF- 4 in both group of patients, indicating that the patients in this study were already in hypercoagulable state preoperatively. It might be attributed to preoperative proinflammatory state as suggested by elevated preoperative hsCRP, and the effect of ulinastatin may not be sufficient enough to abrogate postoperative procoagulant changes in patients, who were already in hypercoagulable state preoperatively.

In view of the role of PMNE on coagulation and fibrinolysis [19], intraoperative administration of ulinastatin has been reported to reduce the amount of early postoperative blood loss in major orthopedic surgery [27], while we could not observe any blood conservative effect of ulinastatin in the current study. These differences might be associated with differences in the degree of PMNE activation and coagulation-fibrinolysis balances between the involved patients in both the studies.

The limitations of this study are follows. Generally, intraoperative ulinastatin was administered at doses of 5,000-6,000 $\mathrm{U} / \mathrm{kg}[6,21]$ and 1,000,000 $\mathrm{U}$ has been used as a maximum dose in cardiac surgery using CPB [5], while there is no available report, which states the optimal dose of ulinastatin in OPCAB. The dose of ulinastatin in this study $(600,000 \mathrm{U})$ might not be sufficient to modulate coagulation function in high-risk patients undergoing OPCAB although the chosen dose of ulinastatin in the current trial was effective in terms of attenuation of proinflammatory response in previous studies [5,21]. In addition, we administered ulinastatin after induction of anesthesia and at the commencement of Y-graft construction, since prothrombotic stimulation was supposed to be most intense during coronary anastomoses due to the superimposed influence of systemic inflammatory response and cumulative regional myocardial warm I/R injury. However, considering the relatively short half-life (33 $\mathrm{min}$ ) of ulinastatin [28], there is a possibility that continuous infusion may be more favorable rather than bolus injection. Indeed, with bolus injection followed by continuous infusion, ulinastatin has been reported to decrease postoperative cardiac troponin levels after OPCAB [29].

Approximately $2 / 3$ of the enrolled patients continued their dual antiplatelet therapy within 5 days of surgery and dual antiplatelet therapy was resumed on the first day of surgery in all the patients in the current study. As recent literature suggests ischemic and/or mortality benefit with some degree of platelet inhibition in patients undergoing CABG [30], increasing number of patients presenting for CABG are on continued dual antiplatelet therapy in our daily clinical practice. Although the association of dual antiplatelet therapy with the development of hypercoagulable state remains unclear, it is hypothesized that it may have influenced the activation of the coagulation parameters. However, the proportions of patients taking aspirin or clopidogrel and timing of discontinuing those medications were not different between the control and ulinastatin groups. Moreover, when comparing the TAT, F1+2 and PF-4 levels in patients who discontinued their dual antiplatelet therapy $>5$ days of surgery, similar results were observed in their cases as presented (data not shown). Therefore, the influence of dual antiplatelet therapy should be negligible, if not, at least same in both groups.

In conclusion, intraoperative administration of 600,000 U of ulinastatin did not convey beneficial influence in terms of coagulation, blood loss and the incidence of MI in high-risk patients with elevated preoperative hsCRP values undergoing multivessel OPCAB.

\section{References}

1. Nesher N, Frolkis I, Vardi M, Sheinberg N, Bakir I, Caselman F, et al. Higher levels of serum cytokines and myocardial tissue markers during on-pump versus off-pump coronary artery bypass surgery. J Card Surg 2006; 21: 395-402.

2. Kon ZN, Kwon MH, Collins MJ, Kallam S, Sangrampurkar R, Ozeki $\mathrm{T}$, et al. Off-pump coronary artery bypass leads to a regional hypercoagulable state not detectable using systemic markers. Innovations (Phila) 2006; 1: 232-8.

3. Kon ZN, Brown EN, Grant MC, Ozeki T, Burris NS, Collins MJ, et al. Warm ischemia provokes inflammation and regional hypercoagulability within the heart during off-pump coronary artery bypass: a possible target for serine protease inhibition. Eur J Cardiothorac Surg 2008; 33: 215-21.

4. Nishiyama T, Hanaoka K. Do the effects of a protease inhibitor, ulinastatin, on elastase release by blood transfusion depend on interleukin 6? Crit Care Med 2001; 29: 2106-10.

5. Bingyang J, Jinping L, Mingzheng L, Guyan W, Zhengyi F. Effects of urinary protease inhibitor on inflammatory response during on-pump coronary revascularisation. Effect of ulinastatin on inflammatory response. J Cardiovasc Surg (Torino) 2007; 48: 497503.

6. Nishiyama T, Yokoyama T, Yamashita K. Effects of a protease inhibitor, ulinastatin, on coagulation and fibrinolysis in abdominal surgery. J Anesth 2006; 20: 179-82.

7. de Ferranti SD, Rifai N. C-reactive protein: a nontraditional serum marker of cardiovascular risk. Cardiovasc Pathol 2007; 16: 14-21. 
8. Balciunas M, Bagdonaite L, Samalavicius R, Griskevicius L, Vuylsteke A. Pre-operative high sensitive C-reactive protein predicts cardiovascular events after coronary artery bypass grafting surgery: a prospective observational study. Ann Card Anaesth 2009; 12: 12732.

9. Esmon CT. Crosstalk between inflammation and thrombosis. Maturitas 2004; 47: 305-14.

10. Stepień E, Plicner D, Branicka A, Stankiewicz E, Pazdan A, SniezekMaciejewska M, et al. Factors influencing thrombin generation measured as thrombin-antithrombin complexes levels and using calibrated automated thrombogram in patients with advanced coronary artery disease. Pol Arch Med Wewn 2007; 117: 297-305.

11. Ikeda U, Yamamoto K, Shimada K. Biochemical markers of coagulation activation in mitral stenosis, atrial fibrillation, and cardiomyopathy. Clin Cardiol 1997; 20: 7-10.

12. Aosasa S, Ono S, Mochizuki H, Tsujimoto H, Ueno C, Matsumoto A. Mechanism of the inhibitory effect of protease inhibitor on tumor necrosis factor alpha production of monocytes. Shock 2001; 15: 101-5.

13. Sato Y, Ishikawa S, Otaki A, Takahashi T, Hasegawa Y, Suzuki M, et al. Induction of acute-phase reactive substances during openheart surgery and efficacy of ulinastatin. Inhibiting cytokines and postoperative organ injury. Jpn J Thorac Cardiovasc Surg 2000; 48: 428-34.

14. Inoue K, Takano H. Urinary trypsin inhibitor as a therapeutic option for endotoxin-related inflammatory disorders. Expert Opin Investig Drugs 2010; 19: 513-20.

15. Park KH, Lee KH, Kim H, Hwang SO. The anti-inflammatory effects of ulinastatin in trauma patients with hemorrhagic shock. J Korean Med Sci 2010; 25: 128-34.

16. Okida M, Masako O, Maruya H, Higashi T, Yukaya H. Intraoperative changes in blood coagulation and the effectiveness of ulinastatin during liver resection. J Anesth 1991; 5: 43-7.

17. Takano S, Kimura S, Ohdama S, Aoki N. Plasma thrombomodulin in health and diseases. Blood 1990; 76: 2024-9.

18. Bjork P, Axelsson L, Bergenfeldt M, Ohlsson K. Influence of plasma protease inhibitors and the secretory leucocyte protease inhibitor on leucocyte elastase-induced consumption of selected plasma proteins in vitro in man. Scand J Clin Lab Invest 1988; 48: 205-11.

19. Madoiwa S, Tanaka H, Nagahama Y, Dokai M, Kashiwakura Y,
Ishiwata A, et al. Degradation of cross-linked fibrin by leukocyte elastase as alternative pathway for plasmin-mediated fibrinolysis in sepsis-induced disseminated intravascular coagulation. Thromb Res 2011; 127: 349-55.

20. Conway EM, Rosenberg RD. Tumor necrosis factor suppresses transcription of the thrombomodulin gene in endothelial cells. Mol Cell Biol 1988; 8: 5588-92.

21. Nakanishi K, Takeda S, Sakamoto A, Kitamura A. Effects of ulinastatin treatment on the cardiopulmonary bypass-induced hemodynamic instability and pulmonary dysfunction. Crit Care Med 2006; 34: 1351-7.

22. MacGillivray TE, Vlahakes GJ. Patency and the pump--the risks and benefits of off-pump CABG. N Engl J Med 2004; 350: 3-4.

23. Mariani MA, Gu YJ, Boonstra PW, Grandjean JG, van Oeveren W, Ebels T. Procoagulant activity after off-pump coronary operation: is the current anticoagulation adequate? Ann Thorac Surg 1999; 67: 1370-5.

24. Lee SS, Singh S, Link K, Petri M. High-sensitivity C-reactive protein as an associate of clinical subsets and organ damage in systemic lupus erythematosus. Semin Arthritis Rheum 2008; 38: 41-54.

25. Hedman A, Larsson PT, Alam M, Wallen NH, Nordlander R, Samad BA. CRP, IL-6 and endothelin-1 levels in patients undergoing coronary artery bypass grafting. Do preoperative inflammatory parameters predict early graft occlusion and late cardiovascular events? Int J Cardiol 2007; 120: 108-14.

26. Thachil J. The prothrombotic potential of platelet factor 4. Eur J Intern Med 2010; 21: 79-83.

27. Lee JY, Lee JY, Chon JY, Moon HS, Hong SJ. The effect of ulinastatin on hemostasis in major orthopedic surgery. Korean J Anesthesiol 2010; 58: 25-30.

28. Jonsson-Berling BM, Ohlsson K. Distribution and elimination of intravenously injected urinary trypsin inhibitor. Scand J Clin Lab Invest 1991; 51: 549-57.

29. Wang GY, Qiu HB, Zhan SG, Li LH. Protection of ulinastatin against myocardial injury induced by off-pump coronary artery bypass graft surgery: report of 24 cases. Zhonghua Yi Xue Za Zhi 2007; 87: 2502-4.

30. Montalescot G, Hulot JS, Collet JP. Antiplatelet therapy and coronary artery bypass graft surgery a fallow land. J Am Coll Cardiol 2010; 56: 2003-5. 